\title{
A Note on Chinese, Korean, and Japanese Names
}

Throughout the running text, I have followed the East Asian convention

1 in which the family name precedes the given name. This rule is reversed, however, in all bibliographical information, including that in footnotes, where the names are rendered in Western order. Also by convention, poets and writers are referred to by pseudonym rather than by family name, most notably in the cases of Sōseki and Bashō. 